\title{
Application Research of Digital Campus Construction Based on Intelligent Access for Teaching Resource
}

\author{
Bo Zhu \\ Dongfang College, Shandong University of Finance and Economics, Tai'an Shandong, 271000, \\ China
}

Keywords: Intelligent access, Teaching resources, Digital campus construction, Application.

\begin{abstract}
With the development of information technology and its wide application in the field of education, digital campus construction has become an important standard to measure the educational management level for institutions of higher learning, which are highly valued in the education sectors. Wireless network, as one of the infrastructures for digitized campus construction, and it has become an important problem of digital campus construction process. Start from intelligent devices are popular in college campuses, based on the analysis of intelligent access to teaching resources construction of digital campus application system, hoping to promote the digital campus construction to have a better development.
\end{abstract}

\section{Introduction}

In the current development goals of Digital Campus Construction, in addition to the pursuit of basic administrative office automation functions, but we also want to be able to achieve a wide range of digital management of human resources, financial assets, and provide focus support to related services of teaching. At this stage, with the further development of information technology, the majority of our colleges and universities have established appropriate campus network, which has the basic conditions for the construction of digital campus, it can be combined with the current intelligent digital device on campus, based on intelligence access to teaching resources to strengthen the digital campus construction, to promote campus teaching management level for the continuously for corresponding strengthened and enhanced.

\section{Campus Network and Digital Campus Construction}

In the related study of the current stage, the campus network construction and digitization of and did not form a unified and clear understanding of the different research point to produce a difference of understanding, but these studies campus network and the construction of digital campus concept, understanding the role of other aspects of a certain convergence. First, the campus network. Defining the scope of the concept of the campus network campus-wide space generally prevail, with some leaps and bounds in terms of time. Specifically refers to an advanced educational philosophy and teaching methods to guide and support for campus teaching activities, research activities and student activities to provide a relatively sound management resource sharing platform for the exchange and use of campus-related information to provide intelligent technology support is provided for the joint development office aspects of the work undertaken unity ${ }^{[1]}$. Second, the digital campus. The main campus digital campus is wired and wireless networks as technical support, combined with the use of more advanced communications and software encryption security technology, campus activities in teaching, scientific research activities and student management activities and other relevant information and resources, the use of shared, integrated and comprehensive implementation of the 
integrated management tools. With the relative scientific management techniques and concepts related to teaching resources to achieve the effective development and utilization and, ultimately, the school building into a virtual digital College campus to promote the working efficiency can be significantly improved, and the campus service functions to expand the campus to promote better development in contemporary society.

\section{Intelligent Access to the Campus Network Communications Design}

Intelligent communication network access campus network framework is the more important part, began to be at the bottom of most basic services router platform-related information processing, and use of the network of teachers and students can see the relevant interfaces at the application layer, you can use general IP network socket socket programming development to provide appropriate support for the point to point communication between teachers and students network. But if the campus network in the framework of digital campus construction process used is based on the Ad-hoc communication mode of mobile communication, the network topology is bound to time corresponding changes and adjustments, the entire network link quality and the state is also bound produce changes in continuity. Therefore, in the design phase of the network of communication, it should not affect the uncertainties brought on a full analysis and study, the frame can be working on a relatively stable campus network communications platform [2]. In this process must be appropriate to use some of the underlying network transmission control systems security protocols, and choose to support both teachers and students at the same time the majority of online teaching resources intelligently access, data communication should therefore be on multiple computers and mobile intelligent terminal problems reasonable analysis.

\section{Situation of Current Campus Network Security}

In the current wireless network technology gradually mature background, set the scientific wireless network solutions to further meet the special needs of the campus network digital construction, building on the campus wireless network for the audience on campus to provide more quality network services, need to further expand the coverage of the campus wireless LAN in the current wired network, it can be anytime, anywhere access to the majority of teachers and students. It should be recognized that at present China's wireless network while being widely used, wireless access, there are some security problems facing relatively severe test. Under normal circumstances, the campus network security mechanism used mainly includes the following three types: First, based on the MAC address has been certified, specifically based on the MAC address has been authenticated MAC address filtering, which each associated wireless access point MAC address list can freely use the network to access the user reasonable limits and if a MAC address to implement the corresponding user access control, MAC will be shown in the relevant user's MAC address, then the user will be able to apply for access to the network, but without the user's address is displayed in the list, the user can not access the network [3]. Second, the shared authentication key. For a campus wireless network design and applications, verify the shared key authentication requires scientific wired algorithms in wireless devices and the associated access point, if you master the correct shared key, the key can be by means of a wireless network to access. Third, 802.1X authentication. This certification protocols are professionals called port-based access control terminal, which is primarily a two-layer protocol, in accordance with $802.1 \mathrm{X}$ client software is set to carry out certification requirements, and then open a logical port, start the appropriate the IP DHCP, ultimately, some network access.

It is easy to be seen, most of the current setting of the wireless campus network access points for wireless access no systematic analysis of security issues, lack even the most basic security work, if not based on the MAC address in the course of running the program on a shared secret key authentication, then the use of intelligent terminals on campus to campus wireless network access process, due to the relevant safety access point a certain lack of security measures, will search for a number of wireless access points, and can be accessed directly, relatively easy. But at this point 
unidentified staff if the presence of a wireless network into the campus network, will certainly likely to pose a threat to network security, adversely affect the campus network construction.

\section{Cryptographic Security Program Design, Improvement and Application of Intelligent Access to Teaching Resources}

In computer Internet security encryption security technology is one of the more important part, IEEE offers three security technology for campus wireless local area network resources that includes TKIP, WEP and CCMP, the relevant technical means used mainly focus on the campus wireless LAN E campus wireless network router building measures, as well as review and identification of campus network security technology infrastructure framework campus wireless network. This paper attempts to WEP technology as an example of intelligent access to teaching resources cryptographic security design, improved application specific analysis.

Weird Equivalent Privacy is short for WEP, which is part of WLAN in the form of network security, which in the late 1990s to be included in the IEEE 802.11, and has since become an important part of network security standards. Use WEP WLAN technology enables obtaining a wired network can reach the level of security. Information confidentiality and security maintenance, WEP uses the RC4 stream cipher in the design and application process, and the use of CRC-32 check mode in the actual operation of the data integrity check and maintain data integrity. In standard definition range, WEP capable of 40 keywords provide support, and on this basis, through suppliers WEP standard for the corresponding extension in the expanded version different or even be able to support 128 even 256-bit key. In the WEP system, generally the initialization key will select an associated initialization vector based on a standard 24-digit ${ }^{[4]}$.

In the intelligent access to teaching resources allocation process, WEP is a security protocol campus intelligent wireless networking equipment related to access to resources that are generally used in networking technologies in the encryption method, and to verify information on the campus network transmission network access to information encryption work, and this has a certain encryption mode selectively. Even at this stage the presence in the campus network security protocol WEP intelligent network access to teaching resources, the actual application process certain drawbacks that make them unable to engage in protection for high security needs resources, but more common in today's wireless network equipment, It has a considerable advantage, so the application of technology to enhance WEP digital campus construction has certain research value.

\section{WEP encryption}

In the specific design and application process, step WEB encryption technology as follows: a program in the selected 24-bit initialization vector and a 40-bit WEP encryption key and reasonable connection between the two; the completion of key after the connection is considered as a whole seed value a pseudo-random number generator possessed; the use of a cyclic redundancy check CRC-32 algorithm is not encrypted plain text calculated to produce a relatively complete ICV, and connect to it; uses the RC4 algorithm ICV, not encrypted plain text and the key sequence calculated to produce the ciphertext; the use of IV can be added to the front of the encrypted data and other aspects of the ICV generation wireless MAC frame payload. This completes the work of WEP encryption, the ability to access its initial application in the teaching of intelligent resource construction of digital campus application process.

\section{WEP Decryption}

In a related design and application process WEP decryption step includes the following aspects: the WEP key and initialization vector 802.11 for effective connection, and the result is as the seed value of the pseudo-random number generator; use CR4 algorithm key sequence and ciphertext frame load calculated to produce plain text without encryption processing; get complete decryption of plain text documents and original ICV; the completion decrypted document integrity calculated the new ICV; 
the the original and the new generation of the ICV ICV comparison, the final outcome of the final required to complete the decryption process.

\section{WEP Improvement and Application}

In the whole calculation and application process, WEP key is calculated through the RC4 algorithm, but due to the algorithm itself has some flaws, it can easily lead to key attacks by outsiders. Therefore, in order to further enhance the encrypted document in the course of security, WEP encryption also need to improve, and produce a new generation of key measures to promote better use WEP technology. This paper selects the ECC algorithm specific analysis. ECC key encryption is a calculation of a public nature, less storage space, but the speed is relatively fast, little effect on the demand for broadband. ECC technology has extremely personalized data structure that has been given on a curve arbitrarily selected two points on the curve and appropriate to add two points, then there must also can draw on another point with a curve, its encryption calculation principles on the use of this relatively unique data structure, and application specific encryption process is as follows: In the actual calculation process, an end user A randomly selected from an elliptic curve Ep (a, b), and appropriately select a point on the curve as the calculated point p; A user combine computing needs to select a specific secret key $\mathrm{K}$, and use the existing algorithm to calculate, and ultimately generate a public nature with a key $\mathrm{L}=\mathrm{KP}$; a user will elliptic curve Ep $(\mathrm{a}, \mathrm{b})$ and point $\mathrm{L}, \mathrm{P}$ transmitted to the user at the other end $B$; $B$ in the receiving user a point $M$ expressly Ep (a, b) on the relevant information, this process has to be transmitted is encoded and get a random integer $\mathrm{x}(\mathrm{x}<\mathrm{r})$; $\mathrm{B}$ binding method for calculating a user point $\mathrm{C} 1=\mathrm{M}+\mathrm{xL}$; $\mathrm{C} 2=\mathrm{XP}$ rational calculation; a user in the final after receiving the relevant information, and then the C1-LC2 reasonably calculated, the final results obtained point M [5]. Such combination of ECC technology completes the WEP improvements and make appropriate application process, access to teaching resources for WEP intelligent digital campus building applications based on exerting a positive influence.

\section{Conclusion}

In summary, intelligent access to teaching resources digital campus system construction is a relatively complicated process, the achievement to the basic objectives of digital campus construction requires high technical conditions for support. Therefore, universities should have pay attention to the encryption security to the intelligent access to teaching resources, and actively explore scientific design, improvement and application measures to promote the intelligent access to teaching resources to provide appropriate support for the construction of digital campus system and promote the construction of digital campus to have a better development in contemporary society.

\section{Reference}

[1] Hou Xiaomao. Empirical Research Based on Intelligent Access to Teaching Resources of Digital Campus Construction, Hunan University, 2014.

[2] Li Xian, Liu Feidu. On the Implementation Path to Digital Campus Platform Construction in Vocational Colleges, Education and Research Programs, 2015 (11): 1-1,2.

[3] Zhang Shuying, Tan Yan, Zheng Jiejin, etc. The Design and Implementation of Campus Digital Electronic Student Attendance System, Shenyang Agricultural University (Social Science Edition), 2012,14 (6): 721-723.

[4] Liu Fang. "Digital Campus" Heterogeneous Database Integration Technology Analysis, Value Engineering, 2012,31 (10): 179-180.

[5] Yue Hong. Campus Digital Media Information Platform, Wireless Internet Technology, 2013 (10): 198-200. 